\title{
Integrated Management of Hypermedia Technical Information based on an EXPRESS analysis
}

\author{
E. Rondeau, S. Roth, T. Divoux, E. Gnaedinger \\ Centre de Recherche en Automatique de Nancy (CNRS URA 821) \\ Université de Nancy I \\ BP 239, 54506 Vandoeuvre-lès-Nancy Cedex, France. \\ Tel : (33) 83-91-24-33, Fax : (33) 83-91-23-90, \\ Email : rondeau@cran.u-nancy.fr
}

\begin{abstract}
The integrated management of technical information induces to take into account several aspects as control, maintenance, supervision. Presently, difficulties result from the lack of information accessibility and from the poor "cohabitation" of the applications which manipulate them. In this paper, we propose to integrate the industrial communication system (MMS), the information system (SQL), and the hypermedia documentation system (HTML). This work is based on an unique modelling using EXPRESS language. Thus, we define a transcription methodology from EXPRESS to MMS, and HTML.
\end{abstract}

\section{Keywords}

Information System, EXPRESS, Industrial Communications, MMS, Hypermedia Systems, HTML.

\section{INTRODUCTION}

The difficulties of technical information management result from the use of heterogeneous applications which are often closed or strongly dedicated : maintenance, control, supervision (Comparot, 1994). Our goal is to propose for users, an unified layer which integrates these applications in order to harmonise information access.

Problems relevant to information sharing (redundancy, inconsistency, access speed and easiness) need a global consideration. The networking of databases management systems is often more difficult to realise than expected. The harmonisation is easier to obtain from the 
communication system point of view : the implementation of these databases management systems becomes less important than the interconnection network management of the different information sources.

European projects like CIM-OSA (Vernadat, 1990) define several integration steps in order to reduce the heterogeneity at hardware, communication, application and information levels. CIM-OSA recommends an entity-relationship formalism for database definition and MMS (ISO 9506) standards for the industrial equipment interconnections. Thus, we have defined translation rules from entity-relationship models to MMS syntax (Rondeau, 1995). Both databases and communication system manipulate the same information, because their design is gathered in a common study. Then, we have modelled the information system using the standardised EXPRESS language (ISO 10303-11P11) and we have defined similar rules to MMS, (EXPRESS to SQL translators also exist).

In this paper, we propose a view of "Hypermedia Technical Information System", which is relevant to several criteria (control, maintenance and supervision), and which allows and accesses to any kind of application, from a unique information system modelling. Tools like HTML (Berners, 1994) (based on SGML (ISO 8879)), presently well known in the Internet community, seem to be particularly suitable for the realisation of such an application.

The following approach allows, from informational EXPRESS analysis, to automatically generate the databases (SQL), the industrial communication system (MMS), and the architecture of associated documentary navigation system (HTML meta-structure).

\section{OBJECTIVES}

The problems of information exchanges between various formalisms (at processing and communication levels) have been studied in research programmes such as CALS (Drouin, 1993) (Computer aided Acquisition on Logistic Support) and STEP (Mason, 1993) (Standard for Exchange of Product data). The main results are the SGML standard definition (Standard General Mark up Language) for managing the electronic documents, and the EXPRESS language definition for modelling the information systems. This standards concept allows the use of interchangeable formats, independent of software platforms and communication systems. This principle can be compared to EDI standards (Blooch, 1991) (Electronic Data Interchange).

In an industrial context, the management of heterogeneous information towards LAN (Local Area Network) is possible by using the MMS standard (Manufacturing Message Specification). The different applications such as maintenance, supervision and control have to cooperate and to be integrated for sharing distributed information. But presently the access of these applications is not unified. So we need specific tools for working in a distributed environment (Ruth, 1995). We propose to develop a layer over both communication and information systems, and a navigation interface in order to manage all kind of applications.

The HTML language which is a subset of SGML standards, has been chosen to achieve this interface layer from EXPRESS modelling. The HTML language is standardised and allows the realisation of easy-to-use hypertext interfaces. But models does not exist to represent, generate and validate document structures created from HTML. Thus, we propose to develop a documentary meta-model from an EXPRESS model. The goal of this work is to present different views (structural, functional or informational), for building a hypertext navigation system. 
This navigation system has to permit the access to control maintenance and supervision applications in order to manage static and dynamic data, and eventually to achieve some actions on the system such as starting programs, writing data, changing parameters,... To succeed, we also have to develop different links between MMS, SQL and HTML structures.

In a first time, we describe the different standards we use (EXPRESS, MMS, HTML). Then we detail the gateways between these different conceptual models.

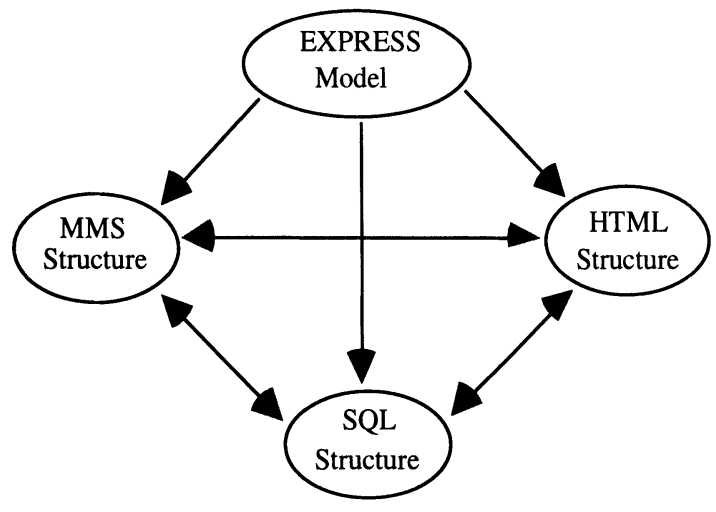

Figure 1 Projection of an EXPRESS model to MMS, SQL and HTML

\section{METHODOLOGY APPROACH}

\subsection{Translation method}

\section{Introduction}

The development of a translator between two description languages has to take into account a set of considerations, such as their respective syntax, the coherence and the semantic retention during their transcoding. Firstly, our objective consists in defining an approach methodology to design a translator, and then, using it, to establish relationships between EXPRESS and MMS and between EXPRESS and HTML. The emergence of such a method needs an overall thought about various translators (especially linked to EXPRESS) according to the decomposition mentioned in (Poyet, 1994). So the study has been focused on two kinds of translators (figure 2) : the first one at implementation level (Morris, 1990) (Jacquelot, 1993) (Yoo, 1993) (Sanderson, 1993); the second one at design level (Griffin, 1993) (Sanderson, 1993) (Bejan, 1993).

A description language can be characterised by its validity domain, which represents its capability for describing the real world. So, the general case study brings up the different kinds of rules allowing to change from a validity domain to another. For that, it is important to specify the translation direction, because the decomposition of the source and target domains are different. Afterwards, we will name DA the source domain and DB the target one. 
A source domain will be divided in three sub-domains (direct, internal, indirect), and a target domain in two ones (direct and indirect). These sub domains are induced by the different rules we describe now. (Figure 3).

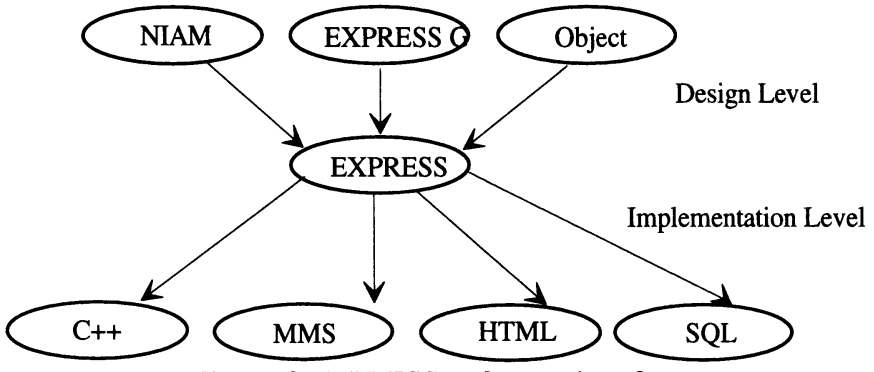

Figure 2 EXPRESS :reference interface.

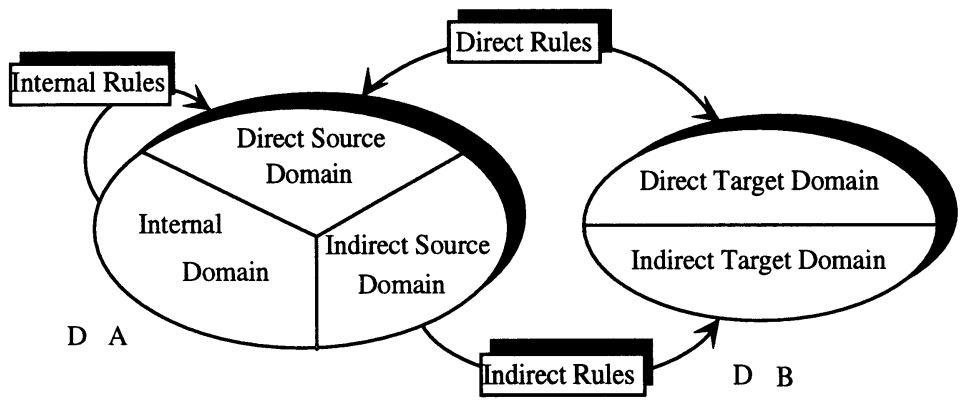

Figure 3 Different kinds of domains and rules.

\section{Rules definition}

The part of a domain which is translatable without semantic losses is called direct domain. Direct rules allow to pass from direct source domain to direct target domain. They are reversible and generally consist in a simple syntax adaptation. Internal rules are defined inside the source domain, in order to obtain a simplified model in the same description language. The objective is to apply direct rules on the simplified model. This simplification induces semantic losses. But it is possible to clearly identify them. Thus, the model designer will know the constraints which are involved by the target domain, during the source domain modelling. So, the target domain imposes its limits to the source one. That defines the influence constraints. The part of the source domain which has not been translated either by direct rules or via internal rules, is called indirect domain. The indirect rules are semantic translation algorithms (Sanderson, 1993) allowing to change from the indirect source domain to indirect target domain. Nevertheless, the whole of semantic is not translated. That defines new influence constraints.

\section{Conclusions}

This method is based on the approach methodology defined in the different translators previously referenced, like the translator between EER formalism and MMS presented in 
(Rondeau, 1994). During the analysis, the translation direction has to be imposed. If we wish a reversible conversion between the domains, it is required to realise two studies, considering domains as source, then as target. This allows to express both internal and indirect rules for each domain. To note the direct rules which are reversible are described once only.

EXPRESS is not the only language available to model applications. Many others different software are more adapted methods or tool boxes and easier to use. Theses methods have been developed in relation with different application fields that need them. In a concurrent engineering context, all these jobs must be able to communicate with each other, thanks to a consensual method. EXPRESS can become an universal language if it is considered as an essential reference interface (Figure 2).

\subsection{EXPRESS/MMS Translator (Divoux, 1994)}

\section{Introduction}

The overall objective of our study consists in defining a set of rules allowing to configure the communications of the different manufacturing equipment's from the information system analysis. The main recommendations to achieve this translator are : to lose no data, to avoid redundant data, to identify the semantic losses all along the modelling.

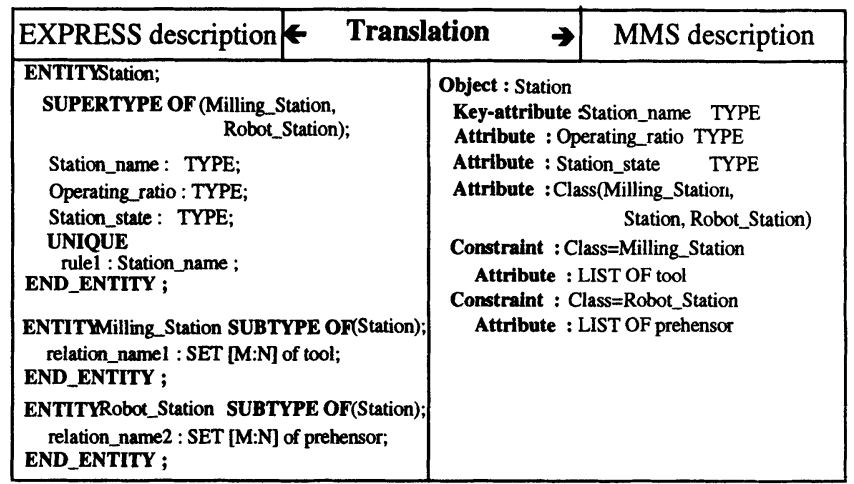

Figure 4 EXPRESS/MMS translation example

\section{Translation rules}

The first step is to define the set of the direct rules between EXPRESS and MMS. These rules identification shows that MMS domain is completely included in EXPRESS one. Six direct (reversible) rules have been defined. They allows to translate each MMS expression in a dual EXPRESS one. EXPRESS is wider than MMS. So, the application of direct rules from an EXPRESS modelling requires to simplify the initial description by using indirect rules. At this time, we are working on their definition.

\section{Translation example}

The following example (figure 4) shows a simplified model of a manufacturing station designed by using EXPRESS language : a station may be either a milling station or a robot 
station. Each kind of machine is described by specific features such as tools for a milling station and prehensors for a robot. The use of the translation rules we have defined leads to an MMS representation which is directly implemented in order to configure the manufacturing equipment making possible their interconnection in an heterogeneous environment. Note on this example that the EXPRESS/MMS translation is reversible since we have only applied direct rules.

\subsection{EXPRESS/HTML Translator}

\section{Introduction}

An EXPRESS/HTML translator is based on the same information system analysis as the EXPRESS model, in order to generate the architecture of the HTML hypertext document. A set of rules is needed to translate the EXPRESS language into an HTML document structure. All these rules are still developed, but the distributed hypertext rules have been already defined.

\section{Translation rules}

First of all, the hypergraph structure is described. It consists of links generation between the different HTML pages. The HTML reference links are then described. These links are useful to send MMS requests to a robot for example.

A keyword class is defined using command words of the EXPRESS language. A list of HTML reference links (Cf. HREF) is then produced as the direct translation of the previous list. With both lists, a set of translation rules is then established. That way, a 'Programmable Logic Translator' is designed.

All these elements are stored in a specific database which can be called the 'HTML document dictionary'. EXPRESS keywords, like 'SUPERTYPE' and 'SUBTYPE', define a structural navigation in a HTML document. And for example, 'DERIVE' or 'FUNCTION' entities can specify an HTML functional navigation.

\section{Translation example}

In our example (Figure 5) the translation of 'SUPERTYPE' and 'SUBTYPE' entities into an hypergraph structure is described. The HTML translation is easily found from an EXPRESS document.

\subsection{MMS/HTML gateway}

\section{Introduction}

The main objective of this gateway is to allow a direct access to MMS services from an HTML page. So, MMS requests can be used through a HTTP server. This study is based on the definition of an HTTP programme interface allowing MMS services invocation. Nevertheless, such interfaces already exist for SQL requests in order to retrieve data from a relational database in a HTML form page.

The local Web server establishes a connection with an equipment and sends HTTP requests including MMS syntax. This interface is based on extended CGI functions (common gateway 
interface). A set of new CGI functions has to be developed in order to realise a gateway between a set of HTML commands and a set of MMS requests.

A dedicated directory gathers the whole CGI functions added to the Web Server (Cf /cgibin/). In such a directory in Internet Server, many others interface programs can be found as : archie, calendar, date, finger, image map, query, uptime, post-query, test-cgi, wais.pl, ...

\begin{tabular}{|c|c|c|c|c|}
\hline EXPRESS Description & Translation & $\rightarrow$ & HTML graph Description & HTML source code \\
\hline \multicolumn{2}{|c|}{$\begin{array}{l}\text { ENTITY Station; } \\
\text { SUPERTYPE OF (Milling_Station, } \\
\text { Robot_Station); } \\
\text { Station_name : TYPE; } \\
\text { Operating_ratio : TYPE; } \\
\text { Station_state : TYPE; } \\
\text { UNIQUE } \\
\text { rule1 : Station_name; } \\
\text { END_ENTITY; } \\
\text { ENTITYMilling_Station SUBTYPE OF(Station); } \\
\text { relation_name1 : SET [M:N] of tool; } \\
\text { END_ENTITY; } \\
\text { ENTITY Robot_Station SUBTYPE OF(Station); } \\
\text { relation_name2 : SET [M:N] of prehensor; } \\
\text { END_ENTITY; }\end{array}$} & Station & {$\left[\begin{array}{l}\text { Station_name } \\
\text { Operating_ratio } \\
\text { Station_state }\end{array}\right.$} & 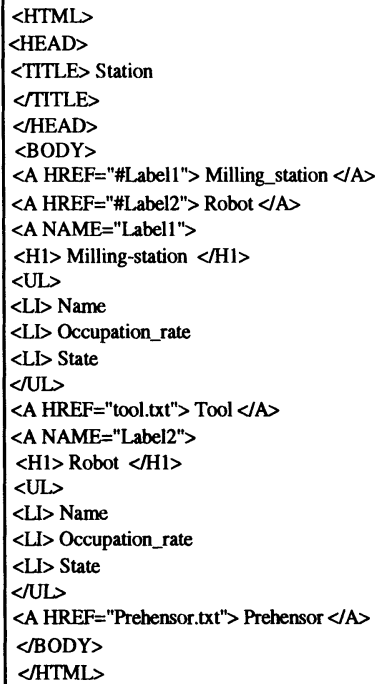 \\
\hline
\end{tabular}

Figure 5 EXPRESS/HTML translation

\section{Example (figure 6)}

In an industrial context, a PC can control a Robot equipment by using MMS services for example on an Ethernet network. These services allow to achieve remote operation such as start a robot task, verify the robot status,... The same PC can also query a Web server with a Netscape or Mosaic software. So the PC station can obtain constructor information on this robot, which is located at the Research department of the robot constructor.

A second PC station (maintenance PC) located outside the local environment, can dialogue on Internet with the robot from a Web server. This server is a gateway between MMS and HTML environments. So the remote maintenance station can observe the robot status or reset it.

\section{CONCLUSION}

In a first time, we just had an informational goal. From an unique EXPRESS conceptual modelling, we wish to be able to decline several implementation of SQL, MMS and HTML physical models. At this first step, the documentary aspect consists in providing an electronic structure, associated to the system in order to inform or to train the user. A dialogue with operators could be added in defining classical HTML forms. The evolution of HTML and new 
technologies providing extensions to WEB servers (Gnaedinger, 1995) will give standard solutions for the publication of dynamic information issued from other applications, or for a direct to relational databases. Nevertheless, we will have to develop our own MMS interface. On another hand, we have to take into account security and accessibility problems, in order to finally provide a real interface for a distributed and documented supervision.

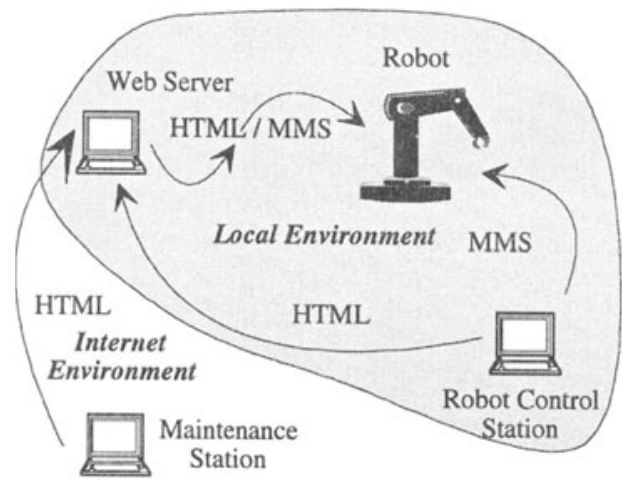

Figure 6 Application example.

\section{REFERENCES}

Bejan A. Wermelinger M.(1993) Semantic Modeling in CIM using EXPRESS and Conceptual Graphs, Third annual EXPRESS User's Group. Berlin, Germany.

Berners-Lee, T., D. Connolly and K. Muldrow (1994) HyperText Markup Language (HTML) version 2.0. - Request for comments internet (ftp://www.ics.uci.edu/pub/ietf/html/ htmlspec2.ps.gz)

Bloch S. (1991) EDI : Echange de données informatisé. Introduction à l'échange de données structuré en syntaxe EDIFACT, éditions Eyrolles.

Comparot-Poussier, C. and C. Christment (1994) Hyperbase for electronic technical document management, Engineering of Information Systems (Ingénierie des systèmes d'information), 2, (5), pp. 533-570, Hermès.

Divoux T., Duchêne D., Rondeau E. (1994) Using EXPRESS to define MMS manufacturing communication, 4th annual EXPRESS User Group International Conference. Greenville, USA.

Drouin, B. (1993) CALS : An essential Strategy for paperless interchange of technical information for manufacturing - current and future standards, Proc. of Computers in Design, Manufacturing and production, 7th Annual European Computer Conf., p.73-78, Paris.

Gnaedinger E., Thiriet J.M., Roth S., Robert M. (1995) Integrated communication architecture to inform citizens about urban services with Telematic Application : Proposition for an urban information system, Networking entities, TEI institut of Piraes, Athens. 
Griffin C.M., Kendall J.M.P. (1993) A Framework For An Advanced NIAM To EXPRESS Converter, Third annual EXPRESS User's Group. Berlin, Germany.

Jacolot C. (1993) Chaîne de développement EXPRESS - C++ - ONTOS, Rapport de DEA Informatique. IFSIC Université de Rennes I.

Leung Ruth, F., C. Leung Horris and F. Hill John (1995) Distributed quality manual via hypermedia, AIAI Conference '95, Nancy.

Mason, H. (1993). Product data representation and exchange -STEP part 1 : overview and fundamental principles, ISO / TC184 / SC4 Status IS, November 20th.

Morris K. (1990) Translating, Express to SQL : A User's Guide, Nationnal PDES Testbed Report Series.

Poyet P. (1994) tools and services for modelling, implementing and exchanging information with the STEP standard, Xstep Interest Group.

Rondeau E., Divoux T., Lepage F., Veron M. (1994) Creation of Virtual Manufacturing Devices by means of information system models. Towards World Class Manufacturing 1993, Phoenix (Arizona). Elsevier Science B.V. (North Holland). 1994 IFIP, pp 259-273.

Rondeau E., Divoux T., Lepage F., Veron M. (1995) MMS Virtual Manufacturing Devices generation: The Paris subway example, Integrated Manufacturing Systems Engineering, P. Ladet, F.B. Vernadat (éditeurs), Chapman \& Hall on behalf of the International Federation for Information Processing IFIP TC5.

Sanderson D., Spooner D. (1993) Mappings between EXPRESS and the Extented Entity Relationship Model, Departement of Computer Science, Technical Report.

Sanderson D., Spooner D. (1993) Mappings between EXPRESS and Traditional DBMS Models, Third annual EXPRESS User's Group. Berlin, Germany.

Totland T. (1990) Translation of Definition and Data from EXPRESS to C++, Dimpola thesis, Norwegian Institute of Technology. Trondheim, 1990.

Vernadat F.B. (1990) Modelling and analysis of enterprise information systems with CIMOSA, Computer Integrated Manufacturing, proceedings of the sixth CIM-Europe annual conference, Lisbon, Portugal, pages 16-27.

Yoo B. , Cha. K.(1993) Checking EXPRESS Constraints in A Heterogeneous Database Environment, Third annual EXPRESS User's Group. Berlin, Germany.

\section{BIOGRAPHY}

E. Rondeau is Associate Professor at the Henri Poincaré - Nancy 1 University (France). He teaches at the Mechanical Engineering Department of the "Nancy - Brabois Institut Universitaire de Technologies".

S. Roth is postgraduated at the Henri Poincare - Nancy 1 University (France). She is teaching at the E.S.S.T.I.N. (Ecole Superieure des Sciences et Technologies de l'Ingenieur de Nancy) an Engineering Studies School in Nancy.

T. Divoux is Associate Professor at the Henri Poincare - Nancy 1 University (France). He teaches at the Telecommunications and Networks Department of the "Nancy - Brabois Institut Universitaire de Technologies".

E. Gnaedinger is Associate Professor at the Henri Poincaré - Nancy 1 University (France). $\mathrm{He}$ teaches at at the E.S.S.T.I.N. (Ecole Superieure des Sciences et Technologies de l'Ingenieur de Nancy) an Engineering Studies School in Nancy. 